TITLE:

\title{
Superconducting transition in disordered granular superconductors in magnetic fields
}

\author{
$\operatorname{AUTHOR}(\mathrm{S}):$ \\ Ikeda, R
}

CITATION:

Ikeda, R. Superconducting transition in disordered granular

superconductors in magnetic fields. PHYSICAL REVIEW B 2006, 74(5):

054510.

ISSUE DATE:

2006-08

URL:

http://hdl.handle.net/2433/49976

RIGHT:

Copyright 2006 American Physical Society 
PHYSICAL REVIEW B 74, 054510 (2006)

\title{
Superconducting transition in disordered granular superconductors in magnetic fields
}

\author{
Ryusuke Ikeda \\ Department of Physics, Kyoto University, Kyoto 606-8502, Japan \\ (Received 15 October 2005; revised manuscript received 28 April 2006; published 24 August 2006)
}

\begin{abstract}
Motivated by a recent argument that the superconducting (SC) transition field of three-dimensional (3D) disordered superconductors with granular structure in a nonzero magnetic field should lie above $H_{c 2}(0)$ in low $T$ limit, the glass transition [or, in two dimensions (2D), crossover] curve $H_{g}(T)$ of disordered quantum Josephson junction arrays is examined by incorporating SC fluctuations. It is found that the glass transition or crossover in the granular materials can be described on the same footing as the vortex-glass transition in amorphouslike (i.e., nongranular) materials. In most of 3D granular systems, the vanishing of resistivity on cooling should occur even above $H_{c 2}(0)$, whereas the corresponding sharp drop of the resistivity in the 2D case may appear only below $H_{c 2}$ as a result of an enhanced quantum fluctuation.
\end{abstract}

DOI: 10.1103/PhysRevB.74.054510

PACS number(s): 74.25.Dw, 74.40.+k

\section{INTRODUCTION}

Throughout extensive studies on phase diagrams of nongranular systems, ${ }^{1-3}$ it is understood at present that the superconducting transition, characterized by the vanishing of resistivity, in homogeneously disordered (amorphouslike) type II superconductors under nonzero fields occurs as a glass transition. As far as the static disorder being pointlike, the resulting glass transition curve $H_{g}(T)$ at nonzero temperatures $(T>0)$ does not deviate much from the melting transition line of a clean vortex lattice. For amorphouslike (nongranular) three-dimensional (3D) systems, the $H_{g}(T)$ curve, determined resistively, approaches a field near $H_{c 2}(0)$ (Ref. 4) in low $T$ limit, even if it apparently approaches a field range below $H_{c 2}(0)$ (Ref. 5) upon cooling as the reduced temperatures $T / T_{c 0}$ is not low enough. On the other hand, it is believed that the glass transition in two-dimensional (2D) disordered case occurs only ${ }^{1,6}$ at $H_{\mathrm{SI}}=H_{g}(T=0)$ below $H_{c 2}(0)$ and corresponds to a field-tuned superconductor-insulator (SI) transition at $T=0 .{ }^{6-8}$

However, it is unclear whether this picture also holds in granular systems or not. By neglecting effects of vortex pinning and superconducting (SC) fluctuations, a glass phase peculiar to disordered granular superconductors was obtained $^{9}$ as a phase lying above $H_{c 2}(T)$ defined $^{10}$ at longer scales than the intergrain spacing (see Fig. 1). Hereafter, this glass phase, which may appear even in $H=0$ separately from

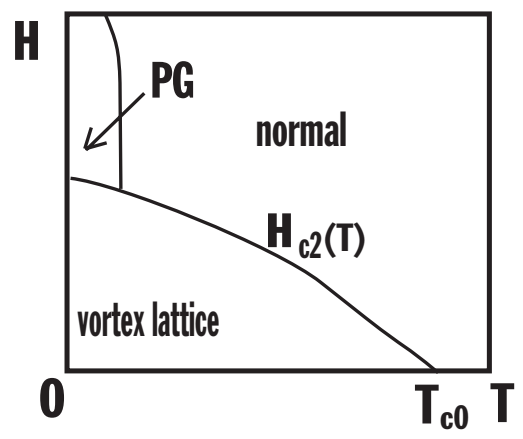

FIG. 1. Mean-field H-T phase diagram (Ref. 9) of a disordered granular superconductor. the Meissner state, will be called the phase glass (PG). However, the fate of PG is not clear once SC fluctuation and vortex pinning effects are taken into account to describe real systems. It was argued recently within a standard model with dissipative quantum phase dynamics that $H_{g}(T)$ of 3D disordered granular systems near $T=0$ should lie far above $H_{c 2}(0)$ (Refs. 11 and 12) and that, even in 2D granular systems with no genuine glass transition ${ }^{1}$ in $T>0$, the corresponding crossover line $H_{g}(T)$ defined from a sharp drop of the resistance should show, on cooling, a divergent (upward) low $T$ behavior as if it terminates at a field $H_{g}(0)$ higher than $H_{c 2}(0)$ (Ref. 11) in low $T$ limit (see Fig. 2). This argument may be consistent with the presence of PG in the mean field phase diagram Fig. 1 if the PG is superconducting. However, if so, it is unclear how the SC (glass) phase and the portion of $H_{g}(T)$ in $H<H_{c 2}$ in Fig. 2 are described. Furthermore, the argument in Ref. 11 for the 2D case is incompatible with the field-tuned S-I transition behavior, which is believed to occur below $H_{c 2}(0)$ even in granular SC thin films. ${ }^{13}$

In this paper, the glass transition curve $H_{g}(T)$ of disordered granular superconductors is examined in the mean field approximation but by including SC fluctuations. We show that $H_{g}(T)$ in the granular case is formally obtained in the same manner as the vortex-glass (VG) transition curve in the amorphouslike case and, hence, is the SC transition curve in $3 \mathrm{D}$ systems at which the resistivity vanishes, ${ }^{1,14}$ at least in

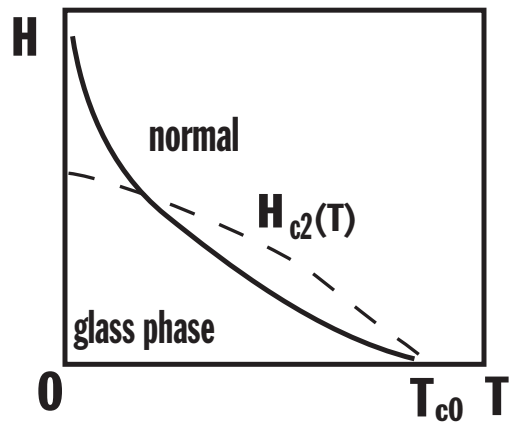

FIG. 2. H-T phase diagram, proposed phenomenologically (Ref. 11), of a disordered granular superconductor. In the 3D case, the resistance vanishes on $H_{g}(T)$ (solid curve). 
the type II limit. Furthermore, we find that, even if the mean field PG phase is absent even at $T=0$, a phase diagram of the type in Fig. 2 with $H_{g}(0)>H_{c 2}(0)$ is generically obtained in a $3 \mathrm{D}$ case, while the situation in which $H_{g}(0)<H_{c 2}(0)$ is easily reached in a 2D case, reflecting an enhanced quantum fluctuation. We argue that the main origin of $H_{g}(0)$ lying above $H_{c 2}(0)$ is an enhancement of vortex pinning due to the $\mathrm{SC}$ fluctuation.

In Sec. II, a model of a disordered and dissipative Josephson-junction array is introduced and rewritten into an effective action, and $H_{g}(T)$ curves in 2D and 3D cases are derived in Sec. III. For comparison, the VG transition curve in the 3D amorphous case is given in Sec. IV in the same framework as in Sec. III, and related discussions and a summary are given in Sec. V.

\section{MODEL}

We start from the Hamiltonian

$$
\mathcal{H}_{\theta}=\alpha \sum_{j}\left(-\mathrm{i} \frac{\partial}{\partial \hat{\theta}_{j}}\right)^{2}-\sum_{\langle i, j\rangle} J_{i j} \cos \left(\hat{\theta}_{i}-\hat{\theta}_{j}\right),
$$

describing a Josephson junction array with a charging energy $2 \alpha$ on each grain, where the pair of indices, $\langle i, j\rangle$, denotes a nearest-neighbor pair of sites (i.e., grains) and $\hat{\theta}_{j}$ is a phase operator on the $j$ th grain. Below, the model will be extended to a more general one, including effects of possible dissipation on each grain and of electromagnetic fields. The most straightforward method of performing this is to express the model (1) into the corresponding quantum action

$$
\begin{aligned}
\mathcal{S}= & \mathcal{S}_{0}-\int_{0}^{\beta} d \tau \sum_{\langle i, j\rangle}\left(\frac { J _ { i j } } { 2 } \operatorname { e x p } \left\{\mathrm { i } \left[\theta_{i}(\tau)-\theta_{j}(\tau)\right.\right.\right. \\
& \left.\left.\left.-e^{*} A_{\mathrm{ex}, i-j}-e^{*} \delta A_{i-j}(\tau)\right]\right\}+ \text { c.c. }\right) \\
& +\int d \tau \int d \tau^{\prime} \sum_{j} \frac{\nu}{\pi\left(\tau-\tau^{\prime}\right)^{2}}\left\{1-\cos \left[\theta_{j}(\tau)-\theta_{j}\left(\tau^{\prime}\right)\right]\right\}
\end{aligned}
$$

in the unit $\hbar=c=1$, where $\beta=1 / T, e^{*}$ is the Cooper-pair charge, $A_{\text {ext }, i-j}$ denotes the line integral of an external gauge field over the bond $i-j, \delta A_{i-j}(\tau)$ is the corresponding gauge disturbance introduced for obtaining the conductivity in the $i-j$ direction, and

$$
\mathcal{S}_{0}(\theta)=\int_{0}^{\beta} d \tau \sum_{i} \frac{1}{4 \alpha}\left(\frac{\partial \theta_{i}(\tau)}{\partial \tau}\right)^{2}
$$

is the action corresponding to the charging energy, i.e., the first term of Eq. (1).

Note that the dissipative (last) term of Eq. (2) is expressed as

$$
\mathcal{S}_{\mathrm{dis}}=\beta^{-1} \sum_{j} \sum_{\omega} \frac{\nu}{2}|\omega|\left|\Phi_{j}(\omega)\right|^{2},
$$

where $\Phi_{j}(\omega)$ is the Fourier transform of

$$
\Phi_{j}(\tau)=\exp \left[\mathrm{i} \theta_{j}(\tau)\right] .
$$

That is, Eq. (4) is nothing but the familiar dissipative term, written in the phase-only approximation, in the timedependent Ginzburg-Landau model.

The quenched disorder in the system is incorporated into a randomness of $J_{i j}=J_{j i}^{*}$ with a nonzero real mean $J_{0}$, i.e., $\overline{J_{i j}}=J_{0}>0$, and a Gaussian distribution $\overline{\left(J_{i j}-J_{0}\right)\left(J_{j i}-J_{0}\right)}=J^{2}$. These relations may be regarded as being due to a random gauge field $a_{i j}$ defined by $J_{i j}-J_{0} \propto \exp \left(\mathrm{i} a_{i j}\right)$. The free energy $F=-\beta^{-1} \ln Z$ will be expressed in terms of the replica trick as $F=-\beta^{-1}\left(Z^{n}-1\right) / n$ in $n \rightarrow+0$ limit. The averaged replicated partition function $\overline{Z^{n}}$ is given by ${ }^{15}$

$$
\overline{Z^{n}}=Z_{0}^{n}\left\langle\exp \left(-\mathcal{S}_{f}-\mathcal{S}_{g}\right)\right\rangle_{0},
$$

where $Z_{0}$ is the partition function of $\mathcal{S}_{0},\langle\rangle_{0}$ denotes the ensemble average on $\Sigma_{1 \leq a \leq n} \mathcal{S}_{0}\left(\theta^{(a)}\right)$, and

$$
\begin{aligned}
\mathcal{S}_{f}= & -\sum_{a=1}^{n} \sum_{\langle i, j\rangle} \int_{0}^{\beta} d \tau J_{0} \cos \left[e^{*} \delta A_{i-j}(\tau)+e^{*} A_{\mathrm{ex}, i-j}\right. \\
& \left.-\theta_{i}^{(a)}(\tau)+\theta_{j}^{(a)}(\tau)\right]+\int d \tau_{1} \int d \tau_{2} \sum_{j} \frac{\nu}{\left(\tau_{1}-\tau_{2}\right)^{2}} \\
& \times\left\{1-\cos \left[\theta_{j}^{(a)}\left(\tau_{1}\right)-\theta_{j}^{(a)}\left(\tau_{2}\right)\right]\right\}, \\
\mathcal{S}_{g}= & \frac{1}{2} \int d \tau \int d \tau^{\prime} \sum \sum_{a, b} \sum_{\langle i, j\rangle} J^{2} \cos \left\{e^{*}\left[\delta A_{i-j}(\tau)-\delta A_{i-j}\left(\tau^{\prime}\right)\right]\right. \\
+ & \left.\theta_{i}^{(a)}(\tau)-\theta_{i}^{(b)}\left(\tau^{\prime}\right)-\theta_{j}^{(a)}(\tau)+\theta_{j}^{(b)}\left(\tau^{\prime}\right)\right\} .
\end{aligned}
$$

Before proceeding further, $\mathcal{S}_{f}$ will be rewritten in the form ${ }^{16}$

$$
\begin{aligned}
\mathcal{S}_{f}= & \text { const }-d J_{0} \beta^{-1} \sum_{\omega} \sum_{i, a}\left[\Phi_{i}^{(a)}(\omega)\right]^{*}\left(1-\frac{\nu}{2 d J_{0}}|\omega|\right. \\
& \left.+\frac{1}{2 d} \mathbf{D}_{i} \cdot \mathbf{D}_{i}^{*}\right) \Phi_{i}^{(a)}(\omega) \\
\simeq & \text { const }-d J_{0} \beta^{-1} \sum_{\omega}\left[\left(2 d J_{0}\right) / 1+\nu|\omega|\right]^{-1} \sum_{i, a}\left[\Phi_{i}^{(a)}(\omega)\right]^{*} \\
& \times\left(1+\frac{1}{2 d} \mathbf{D}_{i} \cdot \mathbf{D}_{i}^{*}\right) \Phi_{i}^{(a)}(\omega)
\end{aligned}
$$

for the cubic or square lattice in $d$ dimension, where $\Phi_{i}(\tau)$ $=\beta^{-1} \sum_{\omega} \Phi_{i}(\omega) e^{-\mathrm{i} \omega \tau}$. Equation (9) is valid up to the lowest order in $\nu|\omega| / J_{0}$ and the Laplacian $\mathbf{D}_{i} \cdot \mathbf{D}_{i}^{*} /(2 d)$, and $\mathbf{D}_{i}$ is the gauge-invariant gradient on the lattice ${ }^{17}$ accompanied by the gauge field $\mathbf{A}_{\mathrm{ex}}+\delta \mathbf{A}(\tau)$. Then, by introducing the conventional SC order parameter $\psi_{i}^{(a)}(\tau)$ and the glass order parameter $q_{i}^{(a b)}\left(\tau_{1}, \tau_{2}\right)=\left[q^{(b a)}\left(\tau_{2}, \tau_{1}\right)\right]^{*}, \bar{Z}^{n}$ becomes $^{9,15,18}$

$$
\frac{\overline{Z^{n}}}{\overline{Z_{0}^{n}}}=\int \mathcal{D} \psi^{(a)} \mathcal{D}\left(\psi^{(a)}\right)^{*} \mathcal{D} q^{(a b)} \exp \left[-\mathcal{S}_{\text {eff }}(\psi, q)\right],
$$

where 


$$
\begin{aligned}
\mathcal{S}_{\mathrm{eff}}(\psi, q)= & \int d \tau_{1} \int d \tau_{2} \sum_{a, b} \sum_{i} \frac{J^{-2}}{2} q_{i}^{(a b)}\left(\tau_{1}, \tau_{2}\right) q_{i}^{(b a)}\left(\tau_{2}, \tau_{1}\right)+\frac{\beta^{-1}}{4 d} \sum_{\omega} \sum_{i} \sum_{a}\left(1+\frac{\nu}{2 d J_{0}}|\omega|\right)\left|\psi_{i, \omega}^{(a)}\right|^{2} \\
& -\sum_{i} \ln \left\{\left\langleT _ { \tau } \operatorname { e x p } \left[\frac{\sqrt{J_{0}}}{2} \int d \tau \sum_{a} \Phi_{i}^{(a)}(\tau)\left(1+\frac{\mathbf{D}_{i} \cdot \mathbf{D}_{i}^{*}}{2 d}\right)^{1 / 2}\left[\psi_{i}^{(a)}(\tau)\right]^{*}\right.\right.\right. \\
& \left.\left.\left.+\frac{1}{2} \int d \tau_{1} d \tau_{2} \sum_{a, b} \Phi_{i}^{(a)}\left(\tau_{1}\right) \times\left[\Phi_{i}^{(b)}\left(\tau_{2}\right)\right]^{*}\left(1+\frac{\widetilde{\mathbf{D}}_{i} \cdot \widetilde{\mathbf{D}}_{i}^{*}}{2 d}\right)^{1 / 2} q_{i}^{(b a)}\left(\tau_{2}, \tau_{1}\right)+\text { c.c. }\right]\right\rangle_{0}\right\},
\end{aligned}
$$

where

$$
\psi(\tau)=\beta^{-1} \sum_{\omega} \psi_{\omega} e^{-\mathrm{i} \omega \tau},
$$

and $\widetilde{\mathbf{D}}_{i}$ denotes the gauge-invariant gradient on the lattice accompanied by the gauge field $\delta \mathbf{A}\left(\tau_{1}\right)-\delta \mathbf{A}\left(\tau_{2}\right)$. Performing the cumulant expansion in powers of $q^{(a b)}$ and $\psi^{(a)}$ in the logarithmic term, various terms, such as

$$
\begin{array}{r}
\int d \tau_{1} \int d \tau_{2}\left\langle T_{\tau} \Phi^{(a)}\left(\tau_{1}\right)\left[\Phi^{(a)}\left(\tau_{2}\right)\right]^{*}\right\rangle_{0} q^{(a a)}\left(\tau_{1}, \tau_{2}\right), \\
\frac{1}{2} \int d \tau_{1} \int d \tau_{2} \int d \tau_{3} \int d \tau_{4}\left\langle T_{\tau} \Phi^{(a)}\left(\tau_{1}\right)\left[\Phi^{(a)}\left(\tau_{3}\right)\right]^{*}\right. \\
\left.\quad \times \Phi^{(b)}\left(\tau_{4}\right)\left[\Phi^{(b)}\left(\tau_{2}\right)\right]^{*}\right\rangle_{0} q^{(b a)}\left(\tau_{2}, \tau_{1}\right) q^{(a b)}\left(\tau_{3}, \tau_{4}\right),
\end{array}
$$

arise in the resulting Landau action $\mathcal{S}_{\text {eff }}$ appropriate to the ensuing analysis. The average \langle\rangle$_{0}$ is carried out by using $\mathcal{S}_{0}$ or its soft-spin version. ${ }^{19}$ For instance, $\left\langle T_{\tau} \Phi_{i}^{(a)}\left(\tau_{1}\right)\left[\Phi_{i}^{(a)}\right.\right.$ $\left.\left.\times\left(\tau_{2}\right)\right]^{*}\right\rangle_{0}$ becomes $\exp \left(-\alpha\left|\tau_{1}-\tau_{2}\right| / 2\right)$ in the low $T$ limit. Below, it will be replaced by its local limit $4 \delta(\tau) / \alpha$ anywhere except in the lowest-order term in $q^{(a b)}$. Furthermore, the $T$ dependence will be taken into account just in the $|\psi|^{2}$ term because, at least, one of such $T$ dependences is necessary in order to keep a reasonable mean field $H_{c 2}(T)$ line for the $\psi$ field. It will be clear that these simplifications are not essential to the present purpose of addressing the low $T$ phase diagram.

Next, let us write $q^{(a b)}\left(\tau_{1}, \tau_{2}\right)$, by following Read et al., ${ }^{18}$ as $Q^{(a b)}\left(\tau_{1}, \tau_{2}\right)-C \delta_{a, b} \delta\left(\tau_{1}-\tau_{2}\right)$ in order to delete the term $\int d \tau_{1} \int d \tau_{2}\left|Q^{(a b)}\left(\tau_{1}, \tau_{2}\right)\right|^{2}$. By representing spatial coordinates in terms of the continuous coordinates $\mathbf{x}$, we finally obtain the following effective Landau action

$$
\begin{aligned}
t \mathcal{S}_{\text {eff }}(\psi, Q ; \delta \mathbf{A})= & \int \frac{d^{d} \mathbf{x}}{a^{d}}\left[\int \frac{d \tau}{\kappa} \sum_{a}\left(\frac{\partial^{2}}{\partial \tau_{1} \partial \tau_{2}}+r\right) Q^{(a a)}\left(\mathbf{x} ; \tau_{1}, \tau_{2}\right) \mid \tau_{1}=\tau_{2}-\frac{\kappa}{3} \int d \tau_{1} d \tau_{2} d \tau_{3} \sum_{a, b, c} Q^{(a b)}\left(\mathbf{x} ; \tau_{1}, \tau_{2}\right)\right. \\
& \times Q^{(b c)}\left(\mathbf{x} ; \tau_{2}, \tau_{3}\right) Q^{(c a)}\left(\mathbf{x} ; \tau_{3}, \tau_{1}\right)+\frac{u}{2} \int d \tau \sum_{a}\left[Q^{(a a)}(\mathbf{x} ; \tau, \tau)\right]^{2} \\
& \left.+\frac{t a^{2}}{4 d \alpha^{2}} \sum_{a, b} \int d \tau_{1} \int d \tau_{2}\left|\left\{-\mathrm{i} \nabla-e^{*}\left[\delta \mathbf{A}\left(\tau_{1}\right)-\delta \mathbf{A}\left(\tau_{2}\right)\right]\right\} Q^{(a b)}\left(\mathbf{x} ; \tau_{1}, \tau_{2}\right)\right|^{2}\right]+t \widetilde{\mathcal{S}}_{\mathrm{eff}},
\end{aligned}
$$

where

$$
\begin{aligned}
t \widetilde{\mathcal{S}}_{\mathrm{eff}}= & a^{-d} \int d^{d} \mathbf{x}\left\{\sum _ { a } \left(\beta^{-1} \sum_{\omega}\left(d_{\psi}|\omega|\left|\psi_{\omega}^{(a)}\right|^{2}\right)+\int d \tau\left[r_{\psi, 0}\left|\psi^{(a)}(\tau)\right|^{2}+c_{\psi}\left|\frac{\partial \psi^{(a)}}{\partial \tau}\right|^{2}+t \widetilde{a}^{2}\left|\left[-\mathrm{i} \nabla-e^{*} \mathbf{A}_{\mathrm{ex}}-e^{*} \delta \mathbf{A}(\tau)\right] \psi^{(a)}(\tau)\right|^{2}\right.\right.\right. \\
& \left.\left.\left.+\frac{t}{2 \alpha}\left(\frac{u_{R}}{\alpha}\right)\left(\frac{4 J_{0}}{\alpha}\right)^{2}\left|\psi^{(a)}(\mathbf{x}, \tau)\right|^{4}\right]\right)-w_{\psi} \sum_{a, b} \int d \tau_{1} \int d \tau_{2}\left[\psi^{(a)}\left(\mathbf{x}, \tau_{1}\right)\right]^{*} Q^{(a b)}\left(\mathbf{x} ; \tau_{1}, \tau_{2}\right) \psi^{(b)}\left(\mathbf{x}, \tau_{2}\right)\right\} .
\end{aligned}
$$

Here, we have introduced a short-length cutoff $a$, which corresponds to the intergrain spacing. We assume that $a$ is much longer than the coherence length of the host material forming the grains and, hence, that the averaged $H_{c 2}(0)$ of the granu- lar system is lower than the microscopic $H_{c 2}(0)$ of the host material forming each grain. ${ }^{10}$ The $H_{c 2}(0)$ mentioned in Sec. I is nothing but this averaged $H_{c 2}(0)$. This is consistent with the assumption in choosing the phase-only model (1) as a 
starting model that the amplitude of the pair field in each grain be robust.

We note that, although the dissipative term in Eq. (1) is reflected only in the term quadratic in $\psi$ of the effective action, the dynamics of the glass fluctuation $\delta Q^{(a b)}$ also becomes dissipative through the coupling $\left(w_{\psi}\right)$ term between $\psi$ and $Q^{(a b)}$ after integrating over the SC $(\psi)$ fluctuations.

Using the soft-spin version of the zero-dimensional action $\mathcal{S}_{0}$, i.e., a Ginzburg-Landau action corresponding to $\mathcal{S}_{0},{ }^{19}$ we find the coefficients to be given by

$$
\begin{gathered}
t=\frac{\alpha^{3}}{4}, \\
\kappa=2, \\
r=\frac{\alpha^{2}}{4}\left[\left(\frac{\alpha}{2 J}\right)^{2}+1\right]\left[\left(\frac{\alpha}{2 J}\right)^{2}-3\right], \\
\frac{r_{\psi, 0}}{t}=\frac{1}{4 d}-\frac{\alpha u_{R}}{J^{2}}\left(1-\frac{2 J^{2}}{\alpha^{2}}\right), \\
\frac{J_{0} T}{2 \alpha^{2}}-\frac{J_{0}}{4 \alpha}\left[\left(\frac{\alpha}{2 J}\right)^{2}-1\right], \\
c_{\psi}=\frac{J_{0}}{2 \alpha^{3}}, \\
d_{\psi}=\frac{t \nu}{8 d^{2} J_{0}}, \\
\tilde{a}^{2}=a^{2} \frac{J_{0}}{4 d \alpha}, \\
w^{2}=\frac{J_{0} t}{\alpha^{2}} .
\end{gathered}
$$

The coefficient $u_{R}$ denotes the renormalized four-point vertex in the soft-spin version of $\mathcal{S}_{0}$. In low-dimensional cases with $d<2$, the renormalization of the fluctuation in a classical Ginzburg-Landau action is well approximated ${ }^{20}$ by the Hartree approximation in which $u_{R}=0$. Based on this fact, the vertex $u_{R} / \alpha$ of the quantum zero-dimensional action $\mathcal{S}_{0}$ may be assumed to take a (dimensionless) number much less than unity.

Below, the Fourier transform of the glass field $Q^{(a b)}(\mathbf{x})$ is defined, by following Ref. 18, as

$$
\begin{aligned}
Q^{(a b)}\left(\tau_{1}, \tau_{2} ; \mathbf{x}\right)= & q^{(a b)}+\frac{1}{\beta} \sum_{\omega \neq 0} \bar{D}_{\omega} e^{-\mathrm{i} \omega\left(\tau_{1}-\tau_{2}\right)} \delta_{a, b} \\
& +\beta^{-2} \sum_{\omega_{1}, \omega_{2}} \delta Q_{\omega_{1}, \omega_{2}}^{(a b)}(\mathbf{x}) e^{-\mathrm{i} \omega_{1} \tau_{1}-\mathrm{i} \omega_{2} \tau_{2}},
\end{aligned}
$$

where the replica symmetric form

$$
q^{(a b)}=q\left(1-\delta_{a, b}\right)+\bar{q} \delta_{a, b}
$$

is assumed for $q^{a b}$ because, here, we do not study the glass phase below $H_{g}$. Furthermore, we focus hereafter on the situation that $H_{g}$ is approached from the higher temperatures at which $q=0$, and $\bar{q}=\beta^{-1} \bar{D}_{0} .{ }^{18}$ The replica-diagonal component $\bar{D}_{\omega}$ is the Fourier transform of the (imaginary) time correlation between two "spins" $\left(\operatorname{Re} \Phi_{j}, \operatorname{Im} \Phi_{j}\right)$ and, hence, is nonvanishing even above $H_{g}(T)$. It is determined by the variational equation $0=n^{-1} \partial \bar{Z}^{n} / \partial \bar{D}_{\omega}$, or

$$
\kappa \bar{D}_{\omega}^{2}=\kappa^{-1}\left(\omega^{2}+r\right)+u \beta^{-1} \sum_{\omega} \bar{D}_{\omega}-w_{\psi} \beta^{-1}\left\langle\psi_{\omega}^{*}(\mathbf{x}) \psi_{\omega}(\mathbf{x})\right\rangle_{s},
$$

where \langle\rangle$_{s}$ denotes the ensemble and space averages. The physically meaningful solution of Eq. (19) is

$$
\bar{D}_{\omega}=-\kappa^{-1} \sqrt{\widetilde{r}_{\omega}+\omega^{2}},
$$

where

$$
\tilde{r}_{\omega}=r+u \kappa \beta^{-1} \sum_{\omega} \widetilde{D}_{\omega}-w_{\psi} \kappa \beta^{-1}\left\langle\psi_{\omega}^{*}(\mathbf{x}) \psi_{\omega}(\mathbf{x})\right\rangle_{s} .
$$

The minus sign in Eq. (20) is chosen so that a physically correct spin correlation along the (imaginary) time direction is recovered. ${ }^{18}$ We note that the last term of Eq. (21) is nonvanishing in $T \rightarrow 0$ limit.

\section{GLASS TRANSITION LINE}

In this section, we will determine the glass transition field $H_{g}(T)$ of the granular system described by Eqs. (14) and (15). It will be seen below that, on cooling, the glass transition is described as a vortex-glass ordering ${ }^{1,14}$ induced by the coupling between the SC fluctuation $\psi$ and the glass fluctuation $\delta Q^{(a b)}$.

We will focus on the (highest) instability temperature at which the glass fluctuation $\delta Q^{(a b)}$ becomes critical at the Gaussian level when the SC fluctuation is fully incorporated. It corresponds to a mean field glass transition in analogy to the normal to Meissner mean field transition following from the BCS theory. (Note that the quasiparticle in the normal state in the latter corresponds to the SC fluctuation in the former.) For simplicity, we shall identify this mean field glass transition line with $H_{g}(T)$. Furthermore, since our main purpose here is to give a correct answer on $H_{g}(T)$ in the high$H$ and low- $T$ portion of the $H-T$ phase diagram, we will use the lowest Landau level (LLL) approximation for the $\psi$ modes. For the LLL modes, the operation $\left(-\mathrm{i} \nabla_{\perp}-e^{*} \mathbf{A}_{\mathrm{ext}}\right)^{2}$ is replaced by $\left|e^{*}\right| H$, and the number of field-induced vortices may be expressed as the total magnetic flux $H S$ multiplied by $\left|e^{*}\right| / 2 \pi$, where $\sqrt{S}$ is the linear system size.

Under the nonzero $\bar{D}_{\omega}$ given by Eq. (20), the glass fluctuation $\delta Q^{(a b)}$ obeys the following effective action $\delta \mathcal{S}_{\text {eff }}$ $=\delta \mathcal{S}_{Q}+\delta \mathcal{S}_{\psi}$ up to the quadratic order in $\delta Q^{(a b)}$, where 


$$
\begin{aligned}
& \delta \mathcal{S}_{Q}= \frac{\beta^{-2}}{2} \sum_{a, b} \sum_{\omega_{1}, \omega_{2}} a^{-d} \int d^{d} \mathbf{x}\left[J^{-2} a^{2} \nabla \delta Q^{(a b)}\left(\omega_{1}, \omega_{2}\right) \cdot \nabla \delta Q^{(b a)}\left(-\omega_{2},-\omega_{1}\right)\right. \\
&\left.-\frac{\kappa}{t}\left(\bar{D}_{\omega_{1}}+\bar{D}_{\omega_{2}}\right) \delta Q^{(a b)}\left(\omega_{1}, \omega_{2}\right) \delta Q^{(b a)}\left(-\omega_{2},-\omega_{1}\right)-2 \frac{J_{0}}{\alpha^{2}}\left(\psi_{\omega_{1}}^{(a)}\right)^{*} \delta Q^{(a b)}\left(\omega_{1},-\omega_{2}\right) \psi_{\omega_{2}}^{(b)}\right], \\
& \delta \mathcal{S}_{\psi}=\beta^{-1} t^{-1} a^{-d} \int d^{d} \mathbf{x} \sum_{a}\left\{\sum _ { \omega } \left[\left(r_{\psi, 0}-J_{0} \alpha^{-2} t \bar{D}_{\omega}+c_{\psi}\left(\omega^{2}+d_{\psi}|\omega|\right)\left|\psi_{\omega}^{(a)}\right|^{2}+\frac{J_{0} t a^{2}}{4 d \alpha}\left|\left(-\mathrm{i} \nabla-e^{*} \mathbf{A}_{\mathrm{ex}}\right) \psi_{\omega}^{(a)}\right|^{2}\right]\right.\right. \\
&\left.+\sum_{\omega_{i}} \frac{t u_{R} J_{0}^{2}}{2 \beta^{2}}\left(\frac{2}{\alpha}\right)^{4} \delta_{\omega_{1}+\omega_{2}, \omega_{3}+\omega_{4}}\left(\psi_{\omega_{1}}^{(a)} \psi_{\omega_{2}}^{(a)}\right)^{*} \psi_{\omega_{3}}^{(a)} \psi_{\omega_{4}}^{(a)}\right\} .
\end{aligned}
$$

The $u \delta Q^{(a a)} \delta Q^{(a a)}$ term [see Eq. (14)] was dropped from $\delta \mathcal{S}_{Q}$. In fact, this term acts ${ }^{18}$ as an interaction term between $\delta Q \mathrm{~s}$ and, hence, may be neglected at the present stage of focusing on the noninteracting (Gaussian) $\delta Q$ fluctuation. In addition, since $u \alpha \ll \sqrt{r}$ for any $J / \alpha$ values of our interest, this term is quantitatively negligible even in obtaining $\bar{D}_{\omega}^{(d)}$ [see Eq. (21)], where $\bar{D}_{\omega}^{(d)}$ denotes $\bar{D}_{\omega}$ in $d$ dimension. Hence, let us drop this small term consistently hereafter. Then, the $T$ and $H$ dependences in $\bar{D}_{\omega}^{(d)}$ arises primarily from the SC fluctuation.

First, let us give the renormalized $\psi$ fluctuation in order to reasonably describe the field range lower than $H_{c 2}(T)$. Hereafter, the $|\psi|^{4}$ term will be treated, as in the nongranular case, ${ }^{25}$ in the Hartree approximation. The Hartree approximation in LLL may be invoked as an infinite-range limit of a nonlocal Ginzburg-Landau model. ${ }^{21}$ Although the use of the nonlocal model makes the details of vortex positional ordering obscure, ${ }^{22}$ the description of the glass ordering in strongly disordered superconductors is not essentially affected by this procedure. Then, the $\psi$ propagator in LLL is defined in the $2 \mathrm{D}(d=2)$ case by

$$
\beta^{-1}\left\langle\psi_{\omega}^{*} \psi_{\omega}\right\rangle_{s}=h^{(2)} G_{\text {dia }}^{(2)},
$$

where

$$
\begin{gathered}
h^{(2)}=\frac{\left|e^{*}\right| H a^{2}}{2 \pi}, \\
G_{\mathrm{dia}}^{(2)}(\omega)=\frac{\alpha}{J_{0} \mu_{\omega}^{(2)}}, \\
\mu_{\omega}^{(2)}=\frac{\alpha}{J_{0}}\left(\frac{c_{\psi}}{t} \omega^{2}+\frac{d_{\psi}}{t}|\omega|+\frac{r_{\psi, 0}}{t}\right)+\frac{2 \pi h^{(2)}}{8}-\alpha^{-1} \bar{D}_{\omega}^{(2)}+\Sigma^{(2)},
\end{gathered}
$$

and

$$
\bar{D}_{\omega}^{(2)}=-\kappa^{-1}\left(r+\omega^{2}-h^{(2)} \frac{\kappa t}{\alpha \mu_{\omega}^{(2)}}\right)^{1 / 2} .
$$

In the 3D case, the corresponding expressions are given by replacing $G_{\text {dia }}^{(2)}$ with $a \int d k_{z} /(2 \pi) G_{\text {dia }}^{(3)}\left(k_{z}\right)$, where

$$
\begin{gathered}
G_{\mathrm{dia}}^{(3)}\left(k_{z} ; \omega\right)=\frac{\alpha}{J_{0}\left(\mu_{\omega}^{(3)}+k_{z}^{2} a^{2} / 12\right)}, \\
\mu_{\omega}^{(3)}=\frac{\alpha}{J_{0}}\left(\frac{c_{\psi}}{t} \omega^{2}+\frac{d_{\psi}}{t}|\omega|+\frac{r_{\psi, 0}}{t}\right)+\frac{2 \sqrt{3} \pi h^{(3)}}{36}-\alpha^{-1} \bar{D}_{\omega}^{(3)}+\Sigma^{(3)}, \\
\bar{D}_{\omega}^{(3)}=-\kappa^{-1}\left(r+\omega^{2}-h^{(3)} \frac{\kappa t}{\alpha\left(\mu_{\omega}^{(3)}\right)^{1 / 2}}\right)^{1 / 2}
\end{gathered}
$$

and

$$
h^{(3)}=3^{1 / 2} \frac{\left|e^{*}\right| H a^{2}}{2 \pi} .
$$

The self-energies $\Sigma^{(2)}$ and $\Sigma^{(3)}$ due to the interaction $\left(u_{R}\right)$ term are given by

$$
\Sigma^{(2)}=32 \frac{u_{R}}{\alpha}(\beta \alpha)^{-1} h^{(2)} \sum_{\omega}\left(\mu_{\omega}^{(2)}\right)^{-1},
$$

and

$$
\Sigma^{(3)}=32 \frac{u_{R}}{\alpha}(\beta \alpha)^{-1} h^{(3)} \sum_{\omega}\left(\mu_{\omega}^{(3)}\right)^{-1 / 2} .
$$

It is easily verified that, in $J \ll \alpha$, Eqs. (26) and (29) reduce to their results in the $J=0$ case.

Although exactly solving these set of equations for each $d$ is, in general, difficult, it can be performed just at the (mean field) glass transition line $H_{g}(T)$. To define $H_{g}(T)$, let us first rewrite Eq. (22) into an effective action $\delta \mathcal{S}_{\text {eff, } Q}$ consisting only of $\delta Q$. Within the Hartree approximation for the $\psi$ fluctuation, $\delta \mathcal{S}_{\text {eff, } Q}$ in the $3 \mathrm{D}$ case becomes

$$
\begin{aligned}
\delta \mathcal{S}_{\mathrm{eff}, Q} \simeq & \frac{1}{2} \int_{\mathbf{k}} \sum_{a, b} \sum_{\omega_{1}, \omega_{2}}\left|\delta Q_{\mathbf{k}}^{(a b)}\left(\omega_{1}, \omega_{2}\right)\right|^{2} \\
& \times\left[J^{-2} k^{2}-\frac{\kappa}{t}\left(\bar{D}_{\omega_{1}}^{(3)}+\bar{D}_{\omega_{2}}^{(3)}\right)-h^{(2)}\left(\frac{J_{0}}{\alpha^{2}}\right)^{2} v_{\mathbf{k}_{\perp}}\right. \\
& \left.\times \int \frac{d q}{2 \pi} G_{\mathrm{dia}}^{(3)}\left(q ; \omega_{1}\right) G_{\mathrm{dia}}^{(3)}\left(q+k_{3} ;-\omega_{2}\right)\right],
\end{aligned}
$$


where $v_{\mathbf{k}_{\perp}}=\exp \left[-\left(k_{1}^{2}+k_{2}^{2}\right) /\left(2 h^{(2)}\right)\right]$. Then, by focusing on the term with $\omega_{j}=0$ and $\mathbf{k}=0$ in Eq. (34), $H_{g}(T)$ is defined as

$$
-2 t^{-1} \bar{D}_{\omega=0}^{(3)}=\frac{h^{(3)}}{2 \kappa \alpha^{2}}\left(\mu_{0}^{(3)}\right)^{-3 / 2}
$$

in the $3 \mathrm{D}$ case, whereas

$$
-2 t^{-1} \bar{D}_{\omega=0}^{(2)}=\frac{h^{(2)}}{\kappa \alpha^{2}}\left(\mu_{0}^{(2)}\right)^{-2}
$$

in the $2 \mathrm{D}$ case, where $\mu_{0}^{(d)} \equiv \mu_{\omega=0}^{(d)}$. To rewrite these equations more explicitly, $\mu_{\omega}^{(d)}$ at $H_{g}(T)$ will be expressed as $\mu_{0}^{(d)}$ $+\delta \mu_{\omega}^{(d)}$. If using Eq. (26) or (29), it is not difficult to obtain $\delta \mu_{\omega}^{(d)}$ as a function of $\mu_{0}^{(d)}$ and, up to the lowest order in $|\omega|$, it becomes

$$
\delta \mu_{\omega}^{(3)}=2\left(\frac{\mu_{0}^{(3)}}{3+64\left(\mu_{0}^{(3)}\right)^{5 / 2} / h^{(3)}} \frac{d_{\psi} \alpha}{t J_{0}}|\omega|\right)^{1 / 2}
$$

in the $3 \mathrm{D}$ case, and

$$
\delta \mu_{\omega}^{(2)}=2\left(\frac{\mu_{0}^{(2)}}{1+8\left(\mu_{0}^{(2)}\right)^{3} / h^{(2)}} \frac{d_{\psi} \alpha}{t J_{0}}|\omega|\right)^{1 / 2}
$$

in the $2 \mathrm{D}$ case, respectively. The expressions on $\mu_{\omega}^{(d)}$ obtained above imply that, to the lowest order in the Matsubara frequency, the glass fluctuation propagator just at the (mean field) glass transition takes the form

$$
\left\langle\delta Q_{\mathbf{k}}^{(a b)}\left(\omega, \omega^{\prime}\right) \delta Q_{-\mathbf{k}}^{(b a)}\left(-\omega^{\prime},-\omega\right)\right\rangle \simeq\left(k^{2}+|\omega|^{1 / 2}+\left|\omega^{\prime}\right|^{1 / 2}\right)^{-1}
$$

after being rescaled spatially.

Now, by applying the above expressions of $\delta \mu_{\omega}^{(d)}$ to the self-consistent equations on $\mu_{0}^{(d)}$

$$
\mu_{0}^{(2)}=\frac{\alpha}{J_{0} t} r_{\psi, 0}+\frac{2 \pi h^{(2)}}{8}-\alpha^{-1} \bar{D}_{\omega=0}^{(2)}+\Sigma^{(2)}
$$

and

$$
\mu_{0}^{(3)}=\frac{\alpha}{J_{0} t} r_{\psi, 0}+\frac{2 \sqrt{3} \pi h^{(3)}}{36}-\alpha^{-1} \bar{D}_{\omega=0}^{(3)}+\Sigma^{(3)},
$$

we obtain the coupled equations [Eqs. (26), (27), (32), (36), (38), and (40)] in the 2D and [Eqs. (29), (30), (33), (35), (37), and (41)] in 3D cases. The resulting $H-T$ relation for each $d$ is nothing but the $H_{g}(T)$ line.

Typical examples of computed $H_{g}(T)$ lines are shown in Fig. 3 for the 3D case and Fig. 4 for the 2D case, where $h$ $=H / H_{c 2}(0)$, and $T_{c 0}$ is the zero field transition temperature. We have commonly used the parameter values, $2 \pi d_{\psi} J_{0} / t$ $=2 \pi \nu / 32=10$ and $u_{R} / \alpha=1 \times 10^{-3}$, and have changed $j_{0}$ $=J_{0} / \alpha$ and $j=J / \alpha$. In Fig. 3, the pairs of parameters $\left(j, j_{0}\right)$ are $(0.05,0.7)$ (left curve) and $(0.28,0.7)$ (right), whereas in Fig. 4 they were chosen as $(0.003,0.3)$ (left curve), $(0.05$, $0.7)$ (center), and $(0.05,0.3)$ (right). The dashed curve in the $3 \mathrm{D}$ case is the thermal melting line in the clean limit.

In the 3D case, the resistivity should vanish at $H_{g}(T)$ because, as is explained in Sec. IV, the obtained $H_{g}(T)$ is essentially the same as the VG transition field ${ }^{1,14}$ in the amor-

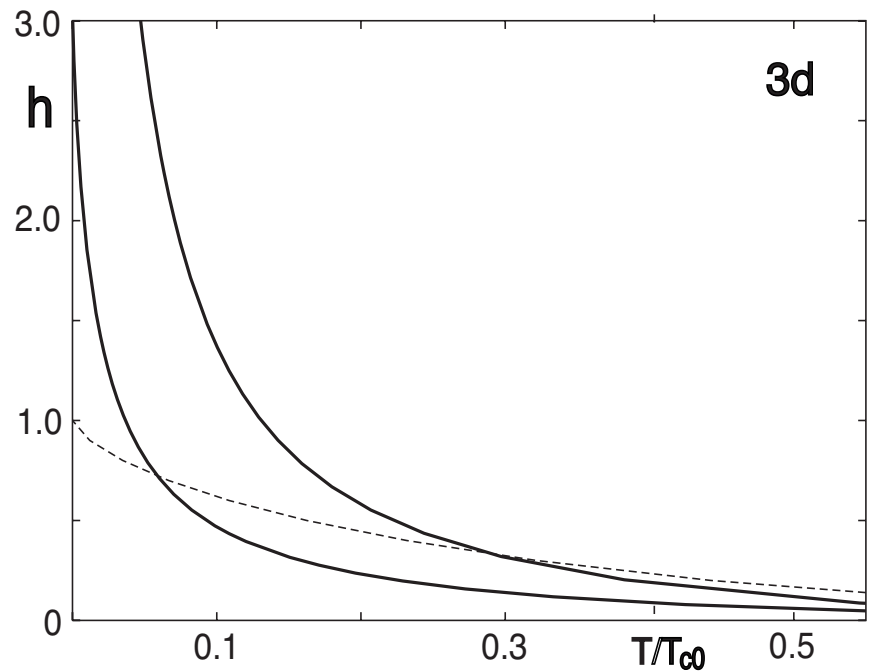

FIG. 3. Examples of $H_{g}(T)$ lines in the 3D case following from the present theory. The $H_{c 2}(T)$ line is given by $h=1-T / T_{c 0}$. See the text regarding the parameter values used for calculations.

phous case. In contrast, $H_{g}(T>0)$ in the $2 \mathrm{D}$ case will be regarded as a crossover line along which the resistivity remarkably drops, ${ }^{11}$ while $H_{g}(0)$ may be identified with the SI transition field $H_{\mathrm{SI}}$ at $T=0$ (see Sec. I).

In the 3D case, the low- $T$ limit $H_{g}(T \rightarrow 0)$ of the glass transition field lies, for most of parameter values we have examined, in $h>1$, i.e., above the mean field $H_{c 2}(0)$. On the other hand, all values of the disorder strength $j$ used in the figures satisfy $\left|\bar{D}_{\omega=0}\right|>0$. This implies assuming that even at $T=0$, the PG order in $H>H_{c 2}(0)$ does not occur. That is, Fig. 3 implies that $H_{g}$ can lie above $H_{c 2}(0)$ more frequently than the prediction from the mean field analysis. ${ }^{9}$ The presence of $H_{g}(T)$ above $H_{c 2}(T)$ at low $T$ is due primarily to the coupling, appearing through $\bar{D}_{\omega=0}^{(d)}$, between the glass field and the SC fluctuation. For brevity, let us imagine that the SC

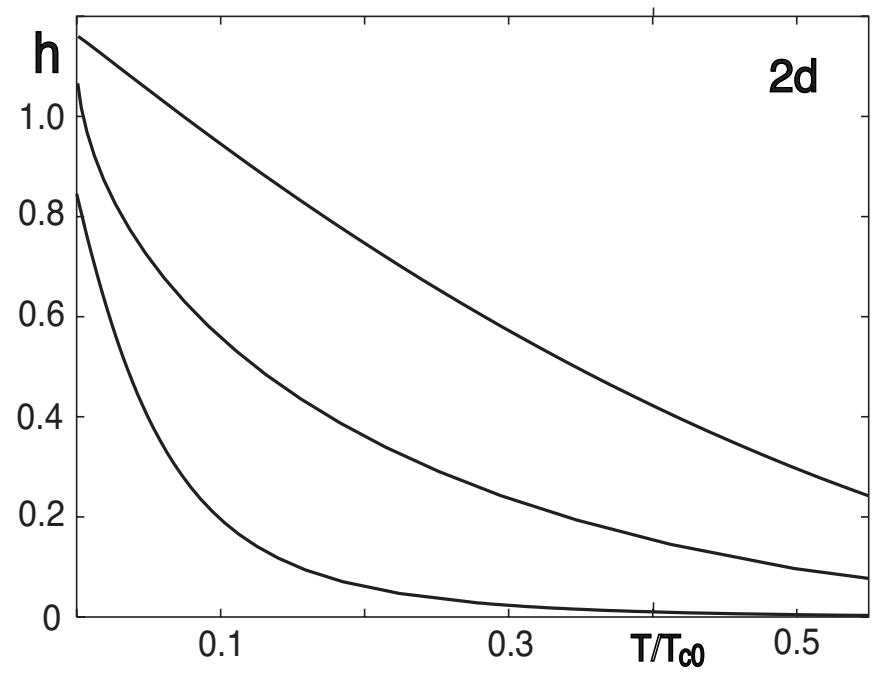

FIG. 4. Examples of $H_{g}(T)$ in the 2D case (sharp crossover lines) comparable to those in Fig. 3. See the text regarding the parameter values used for calculations. 
fluctuation term in $\left(\bar{D}_{\omega=0}^{(d)}\right)^{2}$ (i.e., its second term) will be relatively small in magnitude. Then, from Eq. (35) or (36), $\mu_{0}^{(d)}$ at $H_{g}(T)$ approximately scales like $\left(h^{(d)}\right)^{2 /(6-d)}$. Thus, the second term, $\sim h^{(d)} /\left(\mu_{0}^{(d)}\right)^{(4-d) / 2}$, in $\left(\bar{D}_{\omega=0}^{(d)}\right)^{2}$ grows like $\left(h^{(d)}\right)^{2 /(6-d)}$ with increasing field $h^{(d)}$. As is explained in Sec. $\mathrm{V}$, this implies that the strength of vortex pinning is enhanced by the SC fluctuation with increasing field, although the relation $\mu_{0}^{(d)} \sim\left(h^{(d)}\right)^{2 /(6-d)}$ itself implies a reduction of the SC fluctuation with increasing field above $H_{c 2}$. Such an enhancement of pinning due to the SC fluctuation is peculiar to the granular superconductors at low $T$ and in high fields. In fact, at high $T$ and in weak or intermediate fields, $\mu_{0}^{(d)}$ and, hence, $\left|\bar{D}_{\omega=0}^{(d)}\right|$ rather increases with increasing temperature.

Furthermore, it can be seen from the coupled equations leading to the figures that, in the present quantum model in which the main bare energy scale is not the Josephson coupling $J_{0}$ but the charging energy $\alpha$, the main $J_{0}$ dependence appears in $d_{\psi} \alpha /\left(J_{0} t\right) \propto j_{0}^{-2}$, and hence that a larger $j_{0}$ leads to an enhancement of quantum fluctuation..$^{23,24}$ For this reason, the $(0.05,0.7)$ curve lies below the $(0.05,0.3)$ curve in Fig. 4.

\section{REVIEW OF RESULTS IN NONGRANULAR CASE}

For the purpose of better understanding the content of results in Sec. III, it is useful to compare the results in granular case to those in the nongranular case. ${ }^{14,25}$ Here, we will sketch the corresponding analysis for obtaining the VG transition curve $H_{g}(T)$ of the amorphouslike materials. Within LLL, the familiar GL action derived microscopically takes the form

$$
\begin{aligned}
\mathcal{S}_{G L}[\Psi]= & \int d^{3} r \int d \tau\left(\left\{[\Psi(\mathbf{r}, \tau)]^{*} u(\mathbf{Q} ; \mathbf{r}) \Psi(\mathbf{r}, \tau)\right.\right. \\
& \left.+\xi_{0}^{2}\left|\partial_{z} \Psi(\mathbf{r}, \tau)\right|^{2}+\frac{b}{2}|\Psi(\mathbf{r}, \tau)|^{4}\right\} \\
& \left.+\frac{\gamma}{2 \pi} \int d \tau^{\prime} \frac{\left|\Psi(\mathbf{r}, \tau)-\Psi\left(\mathbf{r}, \tau^{\prime}\right)\right|^{2}}{\left(\tau-\tau^{\prime}\right)^{2}}\right),
\end{aligned}
$$

where $\bar{u}=\ln h, h=H / H_{c 2}(0), \overline{[u(\mathbf{r})-\bar{u}]\left[u\left(\mathbf{r}^{\prime}\right)-\bar{u}\right]}=\Delta_{0} \delta^{(3)}(\mathbf{r}$ $\left.-\mathbf{r}^{\prime}\right), b>0, \gamma>0$, and $\mathbf{Q}$ is the gauge-invariant gradient in directions perpendicular to the applied field $\| \hat{z}$. The role of the random potential leading to the vortex pinning is played by $u(\mathbf{r})-\bar{u}$. The replicated action, arising after the random average, takes the form

$$
\begin{aligned}
\mathcal{S}_{\mathrm{GL}, \mathrm{r}}= & \sum_{a=1}^{n} \mathcal{S}_{\mathrm{GL}}\left[\Psi^{(a)}\right] \\
& -\frac{\Delta_{0}}{2} \int d^{3} r \sum_{a, b} \int d \tau \int d \tau^{\prime}\left|\Psi^{(a)}(\mathbf{r}, \tau)\right|^{2}\left|\Psi^{(b)}\left(\mathbf{r}, \tau^{\prime}\right)\right|^{2} .
\end{aligned}
$$

Alternatively, the last term of $\mathcal{S}_{\mathrm{GL}, \mathrm{r}}$ may be regarded as arising from the action

$$
\begin{aligned}
\mathcal{S}_{\mathrm{GL}, Q}= & \int d \tau \int d \tau^{\prime} \sum_{a, b} \int d^{3} r\left\{\frac{1}{2 \Delta_{0}}\left|\delta Q^{(a b)}\left(\mathbf{r} ; \tau, \tau^{\prime}\right)\right|^{2}\right. \\
& \left.-\left[\Psi^{(a)}(\tau)\right]^{*} \delta Q^{(a b)}\left(\tau, \tau^{\prime}\right) \Psi^{(b)}\left(\tau^{\prime}\right)\right\}
\end{aligned}
$$

after integrating over $\delta Q$. If restarting from the action $\sum_{a=1}^{n} \mathcal{S}_{\mathrm{GL}}\left[\Psi^{(a)}\right]+\mathcal{S}_{\mathrm{GL}, Q}$, we can follow a similar route for obtaining the glass transition line to that in Sec. III. First, the propagator of renormalized $\Psi$ fluctuation in LLL is specified by the Matsubara frequency $\omega$ and, in the 3D case, the wave number $k$ in the direction of the applied field, and given in the form $\left(\mu+\gamma|\omega|+\xi_{0}^{2} k^{2}\right)^{-1}$ in the Hartree approximation. ${ }^{25}$ For weak disorder, the parameter $\mu$ satisfies

$$
\mu=\ln h+b h \xi_{0}^{-3} T \sum_{\omega} \frac{1}{\sqrt{\mu+\gamma|\omega|}},
$$

which corresponds to Eq. (41) in Sec. III. The VG transition field can be defined, just as in Eq. (34), as a critical point of the glass fluctuation $\delta Q$ at which the inverse of the propagator $\left\langle\delta Q^{(a b)}\left(\mathbf{k} ; \omega_{1}, \omega_{2}\right) \delta Q^{(b a)}\left(\mathbf{k} ;-\omega_{2},-\omega_{1}\right)\right\rangle$ vanishes in the limit of vanishing $\mathbf{k}$ and $\omega_{j}$. In the present case, the VG transition line is given by

$$
1=\frac{\Delta h}{2 \mu^{3 / 2}},
$$

which corresponds to Eq. (35) in the granular case, where $\Delta=\Delta_{0} /\left(2 \pi \xi_{0}^{3}\right)$.

In previous works, ${ }^{14,25} \mathrm{Eq}$. (46) was obtained as a pole of the VG susceptibility directly constructed from the action (43). Then, the VG susceptibility takes the same form as the glass fluctuation propagator $\left\langle\delta Q^{(a b)}\left(\mathbf{k} ; \omega_{1}, \omega_{2}\right) \delta Q^{(b a)}(\mathbf{k}\right.$; $\left.\left.-\omega_{2},-\omega_{1}\right)\right\rangle$ in the limit of vanishing $\mathbf{k}$ and $\omega_{j}$. That is, if starting the analysis in Sec. III from an effective action composed only of $\psi$ corresponding to Eq. (43), the glass transition and resistive behavior near $H_{g}(T)$ for the granular case can be described in the same manner as those performed elsewhere. ${ }^{14,25}$ Since the continuous vanishing of resistivity at the VG transition was explained there based on Eq. (43), $H_{g}(T)$ for the 3D granular case in Sec. III has to be also the SC transition line at which the resistivity continuously vanishes.

The VG transition field in the $3 \mathrm{D}$ case and at $T=0$ is determined by the following expression, which is obtained from Eqs. (45) and (46):

$$
\ln h+\frac{\pi^{-1} \xi_{0}^{3} b \omega_{c} h}{(h \Delta / 2)^{1 / 3}+\left[\gamma \omega_{c}+(h \Delta / 2)^{2 / 3}\right]^{1 / 2}}=\left(\frac{h \Delta}{2}\right)^{2 / 3},
$$

where $\omega_{c}$ is a high-frequency cutoff. We note that the fluctuation-corrected (i.e., renormalized) value of $H_{c 2}, H_{c 2}^{(R)}$, is nonzero in $3 \mathrm{D}$ systems at $T=0$ and is given by Eq. (47) with $\Delta=0$. Because of this fact and the $\Delta$ dependence of Eq. (47), the $3 \mathrm{D}$ VG transition field at $T=0$ is always higher than $H_{c 2}^{(R)}(0)$ and approaches $H_{c 2}^{(R)}(0)$ as the disorder $\Delta$ diminishes. ${ }^{7,26}$ In contrast, in the $2 \mathrm{D}$ case, $H_{c 2}^{(R)}(0)$ vanishes and, hence, the VG transition field at $T=0$ (i.e., $H_{\mathrm{SI}}$ ) may lie below the mean field $H_{c 2}(0)$. 


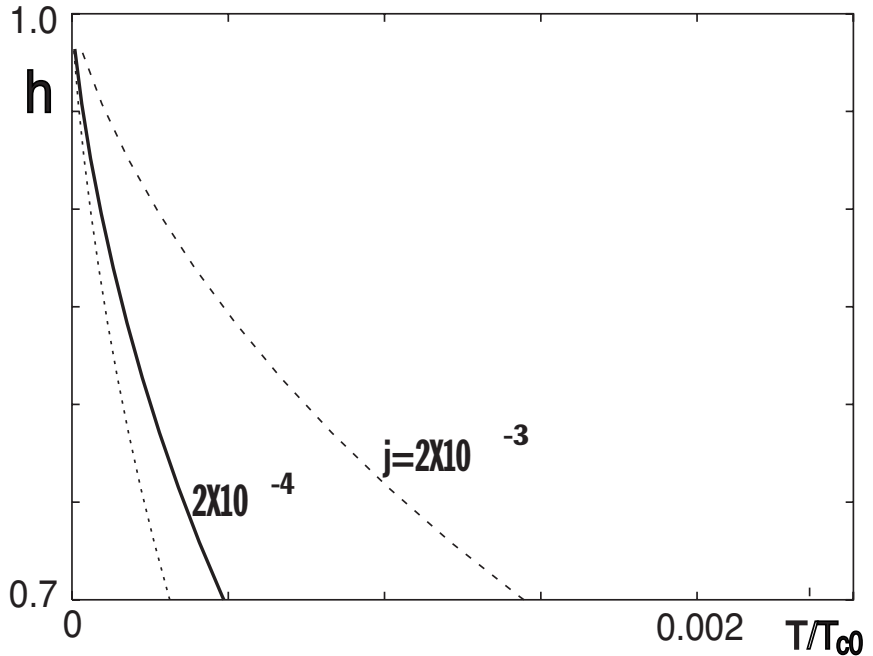

FIG. 5. 3D $H_{g}(T)$ curves at very low $t=T / T_{c 0}$ for the weak disorder cases with $j=1 \times 10^{-4}$ (left), $2 \times 10^{-4}$ (center), and 2 $\times 10^{-3}$ (right). In common, the value $j_{0}=1.5$ was used.

\section{SUMMARY AND DISCUSSIONS}

First, let us start by explaining the 3D $H_{g}(T)$ going beyond the mean field $H_{c 2}$ upon cooling in Sec. III on the basis of the results in Sec. IV for the nongranular case. One reason for the $H_{g}$ growth in higher fields seen in the 3D case is that $H_{g}(0)>H_{c 2}^{(R)}(0)$ in any $3 \mathrm{D}$ case and that $H_{c 2}^{(R)}(0)$ is nonvanishing and tends to lie near the mean field $H_{c 2}(0)$. For instance, even in the left curve in Fig. 3, where $T_{g}(H)$ is significantly lowered, $H_{c 2}^{(R)}(0)$ lies below but close to $H_{c 2}(0)$. For completeness, we show the lowest $T$ behaviors of $H_{g}(T)$ line for extremely low $j$ values in Fig. 5. It shows that, as mentioned below Eq. (47) in the nongranular case, $H_{g}(0)$ approaches $H_{c 2}^{(R)}(0)=0.978 H_{c 2}(0)$ as the disorder $j$ diminishes. That is, since $H_{c 2}^{(R)}(0)$ is the lower limit of the 3D SC (i.e., glass) transition field at $T=0, H_{g}(T \rightarrow 0)$ in $d=3$ lies in $h>1$ in most cases (see also the Appendix ). In contrast, $H_{c 2}^{(R)}(0)$ in the $2 \mathrm{D}$ case is zero and, consequently, $H_{\mathrm{SI}}$ of a system with strong enough quantum fluctuation, as the left curve in Fig. 4 shows, can lie in $h<1$, i.e., much below the mean field $H_{c 2}(0)$. This situation corresponds to the case in which a field-tuned SI transition behavior is seen through resistivity curves in granular materials. ${ }^{13}$

More importantly, in the granular case at low $T$, there is a contribution of the SC fluctuation enhancing the vortex pinning strength with increasing field: As explained in Sec. III, the left-hand side of each of Eqs. (35) and (36) decreases with increasing field due to the SC fluctuation. On the other hand, by comparing Eq. (35) to Eq. (46), the inverse of the left-hand side of Eq. (35), increasing with field, corresponds to a strength of vortex pinning inducing the glass transition. This unfamiliar fluctuation effect, peculiar to granular systems, is quantitatively weakened in the 2D case because the quantum fluctuation is stronger in lower dimensions and selfconsistently increases $\mu_{0}^{(d)}$.

In the present paper, we have examined the low- $T$ behavior of the SC glass transition curve $H_{g}(T)$ of granular super- conductors by applying a theory of quantum spin-glass to the context of superconductivity and have shown that, in contrast to the situations in amorphouslike materials, a situation with $H_{g}>H_{c 2}$ at low $T$ usually occurs in 3D granular systems. This is consistent with the phenomenological picture ${ }^{9,11,12}$ favoring the presence of a superconducting glass in $H$ $>H_{c 2}(0)$. Next, we have shown that, without the PG order, the fluctuation of the glass order parameter plays the role of pinning disorder inducing a vortex-glass instability at $T>0$ in 3D systems under nonzero fields.

Our main message based on the results in Secs. III and IV is that the SC transition in nonzero fields can be described in a single theory, i.e., as a vortex-glass transition, ${ }^{1}$ for both the granular and nongranular (amorphouslike) materials. Because the granular and amorphouslike systems may be continuously connected with each other, e.g., by changing a composition of materials, such a unified view of two limiting models of disordered superconductors should be naturally expected. In a work with a similar purpose to the present one, Galitski and Larkin ${ }^{11}$ have argued that even the SC transition in amorphouslike materials should be described within a model for granular systems. Our result in Sec. III that $H_{c 2}(0)<H_{g}(0)<+\infty$ is consistent with the argument in Refs. 11 and 12. However, our result in the 2D case, given in Fig. 4, that $H_{g}(T \rightarrow 0)<H_{c 2}(0)$ is different from their opinion and rather consistent with experimental facts showing the SI transition behavior.

The vanishing of resistivity on approaching $H_{g}$ from above should imply that the glass phase in $H<H_{g}$ is superconducting, because the transition at $H_{g}$ is continuous in the present case. It is not surprising that the present result disagrees with an argument ${ }^{27}$ based on almost the same model that even the $3 \mathrm{D}$ VG is a metal, because the dissipative term of Eq. (2) was not taken into account correctly in Ref. 27. Without the dissipative term, a finite fluctuation conductivity at finite $T$ above $H_{g}$ cannot be discussed. ${ }^{23}$ In fact, it is difficult to reconcile the metallic response ${ }^{27}$ in $\mathrm{PG}$ phase with the conventional ac Meissner response. ${ }^{28}$ Furthermore, if their model with no Ohmic dissipation ${ }^{27}$ is extended to the case with correlated linelike disorder creating the so-called Bose glass phase, ${ }^{2}$ a (Bose) glass phase with finite resistivity seems to be still obtained in contrast to experimental facts. Our extensive analysis on the conductivity in glass phases will be presented separately. ${ }^{29}$

\section{ACKNOWLEDGMENT}

This work is financially supported by a Grant-in-Aid from the Ministry of Education, Culture, Sports, Science, and Technology, Japan.

\section{APPENDIX}

Here, we estimate $H_{g}(0)$ in 3D amorphouslike case at low $T$ limit by using microscopic parameters in dirty limit. In the ordinary dirty limit where $T \tau \ll 1$, the coefficients in the action (42) are available. ${ }^{23,30,31}$ If reasonably choosing $\gamma \omega_{c}$ to be a constant of order unity, we have $\omega_{c} \sim \tau^{-1}$, where $\tau$ is the elastic scattering time of quasiparticles, and 


$$
\begin{gathered}
b \frac{\omega_{c}}{\xi_{0}^{3}} \simeq\left(\frac{\xi_{0}^{(\mathrm{cl})}}{\xi_{0}}\right)^{3}\left(\frac{T_{c}}{E_{\mathrm{F}}}\right)^{2} \frac{1}{T_{c} \tau}, \\
\Delta \simeq b \frac{\omega_{c}}{\xi_{0}^{3}} \frac{1}{E_{\mathrm{F}} \tau}
\end{gathered}
$$

where $\xi_{0}^{(\mathrm{cl})}$ is the $T=0$ coherence length in clean limit, $T_{c}$ is the zero field transition temperature, and $h$ dependences in the coefficients were neglected by assuming the glass transition field $H_{g}(0)$ in $T \rightarrow 0$ limit to stay close to $H_{c 2}(0)$. This is justified as far as both $b \omega_{c} / \xi_{0}^{3}$ and $\Delta \ll 1$. If $T_{c} \tau \ll 1$, $b \omega_{c} / \xi_{0}^{3} \gg \Delta^{2 / 3}$, and consequently, $H_{g}(0)$ lies below $H_{c 2}(0)$. By contrast, for larger $\tau T_{c}$ values of order unity, $H_{g}(0)$ might lie rather above $H_{c 2}(0)$. Nevertheless, its difference $\left[H_{g}(0)\right.$ $\left.-H_{c 2}(0)\right] / H_{c 2}(0)$ is small according to

$$
H_{g}(0)-H_{c 2}(0) \sim H_{c 2}(0)\left(\frac{T_{c}}{E_{\mathrm{F}}}\right)^{2} \frac{1}{\left(T_{c} \tau\right)^{10 / 3}} .
$$

Of course, when $T_{c} \tau \gg 1$, the right-hand side in Eq. (47) is negligible, and $H_{g}(0)$ becomes $^{7}$ of the order of the nonvanishing $H_{c 2}^{(R)}(0)$ just below the mean field $H_{c 2}(0)$.

In the above analysis, effects of Coulomb repulsion ${ }^{32}$ between quasiparticles were neglected. As in the $2 \mathrm{D}$ case, ${ }^{7,8,30}$ it would play a role of reducing $H_{g}(0)$, although $H_{g}(0)$ does not become lower than $H_{c 2}^{(R)}(0)$ in the $3 \mathrm{D}$ case.

In this manner, the statement in Introduction that $H_{g}(0)$ of nongranular superconductors lies close to $H_{c 2}(0)$ is justified.
${ }^{1}$ D. S. Fisher, M. P. A. Fisher, and D. A. Huse, Phys. Rev. B 43, 130 (1991).

${ }^{2}$ G. Blatter, V. B. Geshkenbein, M. V. Feigel'man, V. M. Vinokur, and A. I. Larkin, Rev. Mod. Phys. 66, 1125 (1994).

${ }^{3}$ T. Nattermann and A. Sheidl, Adv. Phys. 49, 607 (2000).

${ }^{4}$ See Sec. 4 of Ref. 7.

${ }^{5}$ S. Okuma, S. Togo, and M. Morita, Phys. Rev. Lett. 91, 067001 (2003); T. Shibauchi, L. Krusin-Elbaum, G. Blatter, and C. H. Mielke, Phys. Rev. B 67, 064514 (2003); T. Sasaki, T. Fukuda, T. Nishizaki, T. Fujita, N. Yoneyama, N. Kobayashi, and W. Biberacher, Phys. Rev. B 66, 224513 (2002).

${ }^{6}$ M. P. A. Fisher, Phys. Rev. Lett. 65, 923 (1990).

${ }^{7}$ H. Ishida and R. Ikeda, J. Phys. Soc. Jpn. 71, 254 (2002).

${ }^{8}$ R. Ikeda, Phys. Rev. Lett. 89, 109703 (2002).

${ }^{9}$ S. John and T. C. Lubensky, Phys. Rev. B 34, 4815 (1986).

${ }^{10}$ Of course, the microscopic $H_{c 2}$ defined in each grain is much higher than this $H_{c 2}$.

${ }^{11}$ V. M. Galitski and A. I. Larkin, Phys. Rev. Lett. 87, 087001 (2001); 89, 109704 (2002).

${ }^{12}$ V. B. Geshkenbein, L. B. Ioffe, and A. J. Millis, Phys. Rev. Lett. 80, 5778 (1998).

${ }^{13}$ S. Okuma and N. Kokubo, Phys. Rev. B 56, 410 (1997).

${ }^{14}$ R. Ikeda, J. Phys. Soc. Jpn. 65, 3998 (1996); R. Ikeda and K. Myojin, Phys. Rev. B 69, 180505(R) (2004).

${ }^{15}$ T. C. Lubensky, in Ill-Condensed Matter, edited by R. Balian, R. Maynard, and G. Toulouse (North-Holland, Amsterdam, 1979).

${ }^{16}$ Regarding a treatment of an on-site Ohmic dissipative term similar to this; see K-H. Wagenblast, R. Fazio, A. van Otterlo, G. Shon, D. Zappla, and G. T. Zimanyi, Physica B 222, 336 (1996), Sec. IV.
${ }^{17}$ H. Kleinert, Gauge Fields in Condensed Matter (World Scientific, Singapore, 1989).

${ }^{18}$ N. Read, S. Sachdev, and J. Ye, Phys. Rev. B 52, 384 (1995).

${ }^{19}$ See Appendix A of Ref. 18.

${ }^{20}$ D. J. Scalapino, M. Sears, and R. A. Ferrell, Phys. Rev. B 6, 3409 (1972).

${ }^{21}$ S. Hikami and A. Fujita, Phys. Rev. B 41, 6379 (1990).

${ }^{22}$ R. Ikeda, T. Ohmi, and T. Tsuneto, J. Phys. Soc. Jpn. 60, 1051 (1991).

${ }^{23}$ R. Ikeda, Int. J. Mod. Phys. B 10, 601 (1996).

${ }^{24}$ Alternatively, the strength of quantum fluctuation may be tuned by directly reducing $\nu$ with other parameters including $j_{0}$ fixed. Of course, $T_{c 0}$ and $H_{c 2}(0)$ increase with increasing $J_{0}$.

${ }^{25}$ A. T. Dorsey, M. Huang, and M. P. A. Fisher, Phys. Rev. B 45, 523 (1992).

${ }^{26}$ Since we focus here on a narrow field range close to $H_{c 2}(0)$ in clean $3 \mathrm{D}$ systems at $T=0$, effects of the solidification of the vortices occurring in clean systems (see Sec. 4 of Ref. 7) will not be considered here.

${ }^{27}$ J. Wu and P. Phillips, Phys. Rev. B 73, 214507 (2006).

${ }^{28}$ M. V. Feigel'man and L. B. Ioffe, Phys. Rev. Lett. 74, 3447 (1995).

${ }^{29} \mathrm{R}$. Ikeda, cond-mat/0606522 (unpublished).

${ }^{30}$ H. Ishida, H. Adachi, and R. Ikeda, J. Phys. Soc. Jpn. 71, 245 (2002).

${ }^{31}$ R. Sknepnek, T. Vojta, and R. Narayanan, Phys. Rev. B 70, 104514 (2004).

${ }^{32}$ See Ref. 30, and references therein. 